\title{
Randomized, phase II trial of sequential hepatic arterial infusion chemotherapy and sorafenib versus sorafenib alone as initial therapy for advanced hepatocellular carcinoma: SCOOP-2 trial
}

Masaaki Kondo ${ }^{1,2^{*}}$, Manabu Morimoto ${ }^{1,3}$, Satoshi Kobayashi ${ }^{3}$, Shinichi Ohkawa ${ }^{3}$, Hisashi Hidaka ${ }^{4}$, Takahide Nakazawa ${ }^{4}$, Hiroshi Aikata ${ }^{5}$, Takeshi Hatanaka ${ }^{6}$, Daichi Takizawa ${ }^{6}$, Kotaro Matsunaga $^{7}$, Chiaki Okuse ${ }^{8}$, Michihiro Suzuki ${ }^{8}$, Masataka Taguri ${ }^{9}$, Takako Ishibashi ${ }^{10}$, Kazushi Numata', Shin Maeda ${ }^{2}$ and Katsuaki Tanaka ${ }^{1,11}$

\begin{abstract}
Background: The efficacy of hepatic arterial infusion chemotherapy (HAIC) for advanced hepatocellular carcinoma (HCC) remains unclear. We conducted a multi-center randomized phase II study comparing a sequential HAICsorafenib regimen versus sorafenib alone as an initial therapy for HCC.

Methods: Patients were randomly assigned (ratio, 1:1) to receive sequential HAIC with cisplatin followed by sorafenib (HAIC group, $n=35$ ) or sorafenib alone (sorafenib group, $n=33$ ) as an initial therapy. The primary endpoint was the one-year survival rate. Secondary endpoint included overall survival (OS), the 2-year survival rate, the time-to-progression (TTP), the objective response rate (ORR), the disease control rate (DCR), and safety.

Results: For the primary endpoint, the one-year survival rates were $46 \%$ in the HAIC group and $58 \%$ in the sorafenib group. The median OS period was 10.0 months ( $95 \% \mathrm{Cl}, 7.0-18.8)$ in the HAIC group and 15.2 months (95\% Cl, 8.2-19.7) in the sorafenib group (hazard ratio $[\mathrm{HR}], 1.08 ; 95 \% \mathrm{Cl}, 0.63$ to $1.86, P=0.78$ ). The median TTP, ORR and DCR in the HAIC group were 2.8 months $(95 \% \mathrm{Cl}, 1.7-5.5), 14.3$, and $45.7 \%$, respectively, while those in the sorafenib group were 3.9 months $(95 \% \mathrm{Cl}, 2.3-6.8), 9.1$, and $45.5 \%$, respectively. No unexpected adverse events related to HAIC or sorafenib were observed in either group.

Conclusions: Sequential HAIC with cisplatin and sorafenib does not improve the survival benefit, compared with sorafenib alone, when used as an initial therapy for advanced HCC. However, this study was underpowered in regard to its primary and secondary endpoints, so the results should be interpreted with caution.
\end{abstract}

Trial registration: UMIN ID 000006147, registration data: August 11, 2011.

Keywords: Hepatocellular carcinoma, Hepatic arterial infusion chemotherapy, Cisplatin, Sorafenib, Sequential treatment

\footnotetext{
* Correspondence: mkondou@yokohama-cu.ac.jp

${ }^{1}$ Gastroenterological Center, Yokohama City University Medical Center 4-57,

Urafune-cho, Minami-ku, Yokohama, Kanagawa 232-0024, Japan

2Department of Gastroenterology, Yokohama City University Hospital; 3-9,

Fukuura, Kanazawa-ku, Yokohama, Kanagawa 236-0004, Japan

Full list of author information is available at the end of the article
}

(c) The Author(s). 2019 Open Access This article is distributed under the terms of the Creative Commons Attribution 4.0 International License (http://creativecommons.org/licenses/by/4.0/), which permits unrestricted use, distribution, and reproduction in any medium, provided you give appropriate credit to the original author(s) and the source, provide a link to the Creative Commons license, and indicate if changes were made. The Creative Commons Public Domain Dedication waiver (http://creativecommons.org/publicdomain/zero/1.0/) applies to the data made available in this article, unless otherwise stated. 


\section{Background}

Hepatocellular carcinoma (HCC) is one of major causes of death from cancer in the world [1]. On the basis of classification according to the Barcelona Clinic Liver Cancer (BCLC) staging system, patients with intermediate-stage disease showing disease progression after transarterial chemoembolization (TACE) and those with advanced-stage disease have an exceedingly poor prognosis [2, 3]. Sorafenib, an oral multikinase inhibitor, has been demonstrated to improve the overall survival (OS) in advanced HCC patients in two placebo-controlled, randomized phase III studies $[4,5]$. Although sorafenib has been recommended as a first-line treatment not only for advanced-stage HCC patients, but also for intermediate-stage patients who are not expected to benefit from TACE [6], the discontinuation rates because of drug-related adverse events (AEs) were over $40 \%$ in two prospective postmarketing surveillance studies performed in Japan $[7,8]$. Furthermore, the tolerability of sorafenib among elderly HCC patients also seems to be relatively low [9].

Hepatic arterial infusion chemotherapy (HAIC), in which high local concentrations of antitumor agents are achieved with reduced systemic side effects, has been used as a palliative treatment modality. HAIC has been used to treat patients with advanced HCC in Japan and other Asian countries as it has been shown to yield good disease control rates and is tolerated well [10-14]. The CDDP plus 5-fluorouracil (5-FU) $[10,11]$ and 5-FU plus interferon $[12,13]$ regimens are commonly used combination regimens for patients with HCC; however, use of these regimens involves the complex management of the implanted catheter system. However, no prospective or randomized studies have been conducted, and its efficacy remains unclear. HAIC with cisplatin alone, which accounts for $11 \%$ of all HAIC-treated cases of HCC [15] in Japan, is commonly used for treating patients with advanced HCC. Cisplatin is an anticancer agent that acts in a concentration- and time-dependent manner, and it was shown by Court et al. [16] that $48.4 \%$ (range: $34.2-55 \%$ ) of the administered dose is taken up by liver tumors by firstpass kinetics following intravenous injection of ${ }^{195 m}$ cisplatin. Therefore, following selective single-dose administration of cisplatin via the hepatic artery, it is expected that cisplatin would accumulate at even higher concentrations in liver tumors, to exert high therapeutic efficacy. One of the representative HAIC regimens in Japan uses a fine powder formulation of cisplatin (IA call ${ }^{\circ}$ Nippon Kayaku, Tokyo, Japan) adjusted to an approximately three-fold concentration of cisplatin $(1.4 \mathrm{mg} / \mathrm{mL})$ in an aqueous solution, compared with conventional cisplatin formulations $(0.5 \mathrm{mg} / \mathrm{mL})$. The efficacy and tolerability of this formulation were found to be acceptable in patients with unresectable HCC [14].

There are no randomized trials comparing HAIC and sorafenib as a first-line therapy for advanced HCC; therefore, the positioning of HAIC in the treatment of advanced HCC continues to be debated. In this multi-center randomized phase II study, we compared the efficacy and safety of a sequential HAIC treatment using cisplatin followed by sorafenib versus sorafenib alone-a global standard option-as an initial therapy for advanced HCC.

\section{Methods}

\section{Study design and patients}

This randomized, open-label phase II trial was performed at 8 institutes in Japan and was approved by each institute's review board. Written informed consent was obtained from all the patients. This study was performed according to the guidelines of the Helsinki Declaration and the CONSORT guidelines, and was registered with the University Hospital Medical Network Clinical Trial Registry (UMIN ID 000006147).

Eligible patients were diagnosed as having $\mathrm{HCC}$ based on the findings of histopathological examinations, imaging examinations, and/or clinical diagnosis in accordance with the American Association for the Study of Liver Diseases criteria. The eligibility criteria included patients who were unlikely to benefit from surgical resection or locoregional treatment, and were aged 20 years or over, and had a life expectancy of 12 weeks or more, Eastern Cooperative Oncology Group (ECOG) performance status score of 0 or 1 , and a Child-Pugh score of 7 or less. Previous treatment was terminated at least 4 weeks before this study entry. Patients were required to have adequate renal, hematological, and hepatic function, as indicated by a neutrophil count of $10^{3} / \mu \mathrm{L}$ or greater, a platelet count of $50 \times 10^{3} / \mu \mathrm{L}$ or greater, a hemoglobin concentration of $8.5 \mathrm{~g} / \mathrm{dL}$ or more, a total bilirubin concentration of $3.0 \mathrm{mg} / \mathrm{dL}$ or less, a serum creatinine concentration of $1.5 \mathrm{mg} / \mathrm{dL}$ or less, an aspartate and alanine aminotransferase concentration of five-times the upper limit of normal or less, or a serum amylase concentration of two-times the upper limit of normal or less. Patients with extrahepatic metastasis, in whom the lesions were determined by the attending physicians as not being determinants of the prognosis, were allowed to participate in this study. The exclusion criteria were as follows: previous treatment using sorafenib or cisplatin, the presence of serious cardiovascular disease, the presence of esophageal gastric varices and/or gastroduodenal ulcers requiring treatment, the presence of other advanced carcinomas, pregnancy, and extrahepatic lesions which will affect their prognosis.

Patients were randomly assigned in a 1:1 ratio to receive sequential HAIC with cisplatin followed by sorafenib upon progression (HAIC group) or sorafenib alone (sorafenib group) as an initial therapy, using minimization method with a random element using (1) institute, (2) presence of portal vein tumor embolism, (3) presence of extrahepatic lesion. Random number was generated by SAS 9.3 (SAS Institute, Cary, NC, USA). Eligible patients were stratified by the presence or absence of portal vein thrombosis or 
extrahepatic spreading and the institution at which they received treatment. They were enrolled by their doctor in charge and randomization is performed centrally by Data Center, Yokohama City University Medical Center, using Web registration system.

\section{Procedures}

All treatments administered during the trial were open labelled. In the HAIC group, the catheter was inserted into the femoral artery using the Seldinger technique [17] and advanced into the celiac artery; then a microcatheter was advanced into the proper hepatic artery for the chemoinfusion. A fine powder formulation of cisplatin (cisplatin powder) was dissolved in saline solution that had been heated to $50^{\circ} \mathrm{C}$. This solution was then administered at a dose of $65 \mathrm{mg} / \mathrm{m}^{2}$ over a period of about $30 \mathrm{~min}$ [18]. This treatment was repeated at an interval of 4 to 6 weeks until intolerable toxicity or radiological progressive disease (PD). HAIC treatment also halted according to a planned switch criterion-Clinical PD-based on the alpha-fetoprotein (AFP) and des-gamma carboxyprothrombin (DCP) levels. Clinical PD was defined as follows: an increase of $20 \%$ or more in both the AFP and DCP levels compared with the levels at the time of the previous treatment, an increase in the AFP level of $20 \%$ or more of an AFP level equivalent to more than $500 \mathrm{ng} / \mathrm{mL}$ at the time of the previous treatment, or an increase in the DCP level of $20 \%$ or more of a DCP level equivalent to more than $500 \mathrm{mAU} / \mathrm{mL}$ at the time of the previous treatment. These definitions were derived from the AFP and DCP levels of PD patients who achieved SD after the first session of HAIC but exhibited PD after the second session (personal communication). Patients who meet these withdrawal criteria received sorafenib treatment within 14 days after the determination PD. Patients whose HAIC treatments were terminated because of adverse events received sorafenib treatment once their adverse effects had recovered to a grade of less than 1 .

In the sorafenib group, sorafenib was initiated at a dose of $400 \mathrm{mg}$ twice daily. An initial dosage reduction to 400 mg once daily was allowed in some elderly patients or some patients decreasing hepatic reserves. If intolerable toxicities appeared, treatment interruptions and dose reduction were also allowed.

This study protocol was terminated if radiological PD was confirmed after sorafenib treatment in both groups. An attending physician could decide the post-protocol treatment as to whether sorafenib treatment continue, or switch to / use together with other treatment options.

\section{End points}

The primary end point of the study was the one-year survival rate, and the secondary end point were overall survival (OS), the 2-year survival rate, the time to progression (TTP), the objective response rate (ORR), the disease control rate (DCR), and safety. OS was measured from the date of randomization until death or the last consultation day. TTP was measured from the date of the first HAIC session or starting sorafenib treatment until the date of confirmation of the tumor progression radiologically or deterioration of the obvious patients' condition. The tumor response was evaluated on the basis of the modified Response Evaluation Criteria for Solid Tumors (mRECIST) [19], based on dynamic computed tomography $(\mathrm{CT})$ or magnetic resonance imaging (MRI) performed every 4-6 weeks during sorafenib treatment and at the end of 4 weeks after each HAIC session. The toxicities were evaluated based on the Common Terminology Criteria for Adverse Events (CTCAE 4.0).

\section{Statistical analysis}

Efficacy was analyzed in the intent-to-treat population, defined as all randomly assigned patients, and safety was analyzed for all the patients who received each treatment. The sample size calculations were based on the following: with a null one-year survival rate of $43.5 \%$, and an expected one-year survival rate of $57.4 \%$ in patients receiving HAIC with cisplatin followed by sorafenib therapy, to obtain a clinically meaningful improvement in the median OS from 11 months in the sorafenib group to 15 months in the HAIC group with an $80 \%$ power to detect a superior survival outcome in the HAIC group using a one-sided alpha level of $5 \%$, it was determined that 42 patients should be assigned to each group in a random manner. Statistical analyses were performed using SAS 9.3 (SAS Institute, Cary, NC, USA). Continuous parameters were expressed as the mean \pm standard deviation or the medians and ranges, and categorical variables were expressed as the numbers and percentages or the frequencies. The assessment of differences in the baseline features of patients between both groups was determined using the Student $t$-test for continuous variables and the $X^{2}$ test for categorical variables. The statistical significance of differences in the ORR and DCR between the two groups were assessed using the $\chi^{2}$ test for categorical variables. The hazard ratio (HR) and 95\% confidence interval (CI) were determined to estimate the efficacies of the sorafenib and HAIC treatments, respectively. A two-side $P$ value of less than 0.05 was considered statistically significant. Survival curves for OS and TTP were plotted using the Kaplan-Meier method, and any significant differences between the two groups were compared using the log-rank test. Variables that reached a $P$ values of less than 0.05 were regarded as significant in the univariate cox regression analysis.

\section{Results}

Patients and treatment administration

From August 2011 through November 2014, 70 patients were enrolled in this study from 8 institutions. Because 
of slow accrual, this study closed prematurely with a reduced sample size, unlike the planned enrolled number of patients. All the patients were randomly allocated to the HAIC group (36 patients) or the sorafenib group (34 patients). One patient was excluded from each group after they withdrew consent. Follow-up was continued until October 2015, corresponding to 1 year after the enrollment of the last patient. Table 1 shows the baseline characteristics of the patients at the start of this study; the characteristics were evenly balanced between the treatment groups.

In the HAIC group, the median number of HAIC treatments was 2 sessions (range, 1-11 sessions). Tumor-stage improvement was achieved in 4 patients, and they received subsequent conversion options (resection in one patient, RFA in one, and TACE in two patients). Eight patients did not receive subsequent sorafenib treatment because of a deterioration in their general conditions $(n=3)$, a decrease in their hepatic functional reserves $(n=2)$, drug-related toxicities $(n=1)$, a change in treatment to ablation after consultation with the patient $(n=1)$, and the patient's request $(n=1)$. Twenty-three $(66 \%)$ of the 35 patients in the HAIC group received subsequent sorafenib treatment upon radiological $\mathrm{PD}(n=16)$ or clinical $\mathrm{PD}(n=7)$. The median duration between the start of HAIC treatment and subsequent sorafenib treatment was 2.6 months. Eightpatients initially received $400 \mathrm{mg}$ of sorafenib twice daily, while 15 patients received less than $800 \mathrm{mg}$ daily. The median duration of sorafenib treatment was 2.8 months (range, $0.2-16.5$ months), and the median average daily dose (range) was $400 \mathrm{mg}$ (325-800 mg). The study protocol was terminated upon radiological PD $(n=16)$ or drugrelated toxicities $(n=6)$; however, one patient terminated sorafenib treatment upon the achievement of a CR.

In the sorafenib group, the median duration of sorafenib treatment was 2.7 months (range, 0.1-27.3 months). Of the 33 patients, 15 patients initially received $400 \mathrm{mg}$ twice daily, while 18 patients received less than $800 \mathrm{mg}$ daily. The median average daily dose (range) was $400 \mathrm{mg}$ (227-800 mg). The study protocol was terminated upon radiological PD $(n=22)$ or drug-related toxicities $(n=11)$.

\section{Efficacy}

The one-year survival rate, which was the primary end point of the study, was $46 \%$ in the HAIC group, which was not superior to the rate of $58 \%$ in the sorafenib group. The 2-year survival rates were similar: $22 \%$ in the HAIC group, and $24 \%$ in the sorafenib group. The ORR

Table 1 Patients' characteristics in the sorafenib group and the HAIC group

\begin{tabular}{|c|c|c|c|}
\hline Variables & Sorafenib group $(n=33)$ & HAIC group $(n=35)$ & $P$ \\
\hline \multicolumn{4}{|l|}{ Sex } \\
\hline Male/Female & $27 / 6$ & $28 / 7$ & 1.000 \\
\hline Mean age (years) & $70.9 \pm 9.1$ & $72.0 \pm 7.0$ & 0.571 \\
\hline \multicolumn{4}{|l|}{ Cause } \\
\hline $\mathrm{HCV} / \mathrm{HBV} / \mathrm{NBNC}$ & $20 / 4 / 10$ & $21 / 3 / 11$ & 0.308 \\
\hline \multicolumn{4}{|l|}{ Previous treatment } \\
\hline Ablation/TACE+TAE/None & $1 / 20 / 12$ & $2 / 24 / 9$ & 0.308 \\
\hline \multicolumn{4}{|l|}{$\mathrm{BCLC}$} \\
\hline$A / B / C$ & $2 / 13 / 18$ & $2 / 14 / 19$ & 0.994 \\
\hline \multicolumn{4}{|l|}{ UICC } \\
\hline$\|/\| A /\|/\| B / \| C / I V A / I V B$ & $14 / 3 / 7 / 1 / 3 / 5$ & $15 / 2 / 6 / 2 / 4 / 6$ & 0.802 \\
\hline \multicolumn{4}{|l|}{ Child-Pugh class } \\
\hline $5 / 6 / 7$ & $10 / 19 / 4$ & $20 / 11 / 4$ & 0.061 \\
\hline \multicolumn{4}{|l|}{ PVTT } \\
\hline yes / no & $22 / 11$ & $21 / 14$ & 0.621 \\
\hline \multicolumn{4}{|l|}{ Extrahepatic metastasis } \\
\hline yes / no & $8 / 25$ & $10 / 25$ & 0.786 \\
\hline \multicolumn{4}{|l|}{ Gastroesophageal varices } \\
\hline yes / no & $10 / 23$ & $5 / 30$ & 0.099 \\
\hline $\operatorname{AFP}(n g / m L)^{a}$ & $216.7(5-161,160)$ & $67.3(2-281,600)$ & 0.688 \\
\hline $\mathrm{DCP}(\mathrm{mAU} / \mathrm{mL})^{\mathrm{a}}$ & $1068(14-272,000)$ & $335(12-99,800)$ & 0.364 \\
\hline
\end{tabular}


tended to be higher in the HAIC group than in the sorafenib group (14.3\% versus $9.1 \%$, respectively; $P=0.710$; Table 2); however, the DCR was similar: $45.7 \%$ in the HAIC group, and $45.5 \%$ in the sorafenib group. The median OS in the HAIC group was 10.0 months (95\% CI, 7.0-18.8), which was not significantly different from that in the sorafenib group (median, 15.2 months; 95\% CI, 8.2-19.7 months) (HR, 1.08; 95\% CI, 0.63-1.86; $P=0.78$; Fig. 1). The median TTP for the HAIC treatment was 2.8 months (95\% CI, 1.7-5.5 months), while that for the sorafenib treatment in the sorafenib group was 3.9 months (95\% CI, 2.3-6.8 months) (HR, 1.17; 95\% CI, $0.65-2.10 ; P=0.60$; Fig. 2). In the HAIC group, 23 patients received subsequent sorafenib treatment after HAIC failure; the ORR and DCR of the group that received subsequent sorafenib treatment were 4.3 and $34.8 \%$, respectively (Table 2 ), and the median TTP of the group that received subsequent sorafenib treatment was 4.2 months (95\% CI, 2.6-6.5 months).

Subgroup analyses of OS according to the allocation factors, including disease etiology (hepatitis $\mathrm{C}$ or non-hepatitis C), portal vein tumor thrombosis (presence or absence), and extrahepatic metastasis (presence or absence), did not show any superiorities of HAIC to sorafenib between the two groups (Fig. 3); however, in patients with a baseline AFP $\geq 400 \mathrm{ng} / \mathrm{mL}$, the OS of the sorafenib group was significantly better than that of the HAIC group (HR, 2.86; 95\% CI, 1.15-7.10; $P=0.018$ ).

Among the 18 patients with extrahepatic metastasis, 10 were included in the HAIC group and the remaining 8 in the sorafenib group. Of the 10 patients in the HAIC group, the treatment response was rated as PR and SD in 1 and 3 patients, respectively, whereas PD was noted in 5 patients and the treatment effect was not evaluated in 1 patient. On the other hand, of the 8 patients in the sorafenib group, the response was rated as SD in 4 patients, PD was noted in 3 patients, and the effect was

Table 2 Effectivity due to sorafenib treatment in the sorafenib group and the HAIC group

\begin{tabular}{llll}
\hline mRECIST & $\begin{array}{l}\text { Sorafenib } \\
\text { group }(\%)\end{array}$ & HAIC group (\%) \\
\cline { 3 - 4 }$(n=33)$ & HAIC & sorafenib \\
$(n=35)$ & $(n=23)$ \\
\hline CR & $0(0)$ & $0(0)$ & $1(4)$ \\
PR & $3(9)$ & $11(14)$ & $0(0)$ \\
SD & $12(36)$ & $16(46)$ & $7(30)$ \\
PD & $15(46)$ & $3(9)$ & $14(61)$ \\
NE & $3(9)$ & $5(14)$ & $1(4)$ \\
ORR & $3(9)$ & $16(46)$ & $1(4)$ \\
DCR & $15(46)$ & $8(35)$ \\
\hline
\end{tabular}

HAIC hepatic arterial infusion chemotherapy, $C R$ complete response, $P R$ partial response, $S D$ stable disease, $P D$ progressive disease, $N E$ not evaluable, $O R R$ objective response rate, $D C R$ disease control rate not evaluated in 1 patient. The median TTP was 62 days in the HAIC group and 82 days in the sorafenib group, with no significant difference between the two groups $(p=0.945)$.

\section{Safety (Table 3)}

In the HAIC group, the most frequently observed adverse events were an elevated aspartate or alanine aminotransferase level (9\%). One patient (3\%) discontinued HAIC because of unacceptable drug-related toxicities (total bilirubin elevation). In 23 patients who received subsequent sorafenib treatment, six patients (26\%) discontinued sorafenib treatment because of unacceptable drug-related toxicities. The frequently observed adverse events of grade 3 or higher were hypertension (35\%), an elevated aspartate or alanine aminotransferase level (26\%), hand-foot skin reaction (26\%), and an elevated lipase level (17\%).

In the sorafenib group, eleven patients (33\%) discontinued sorafenib treatment because of unacceptable drug-related toxicities. The frequently observed adverse events of grade 3 or higher were an elevated lipase level (27\%), an elevated aspartate or alanine aminotransferase level (21\%), hypertension (18\%), and hand-foot skin reaction (15\%).

\section{Post-protocol treatment}

Post-protocol treatment was given to 18 patients $(51 \%)$ in the HAIC group and 29 patients (88\%) in the sorafenib group (Table 4). Among the patients receiving postprotocol options, the main option ( $\geq 10$ patients) was sorafenib continuation $(29 \%)$ in the HAIC group, while HAIC (55\%) and sorafenib continuation (33\%) were administered in the sorafenib group.

\section{Discussion}

Our study examined whether sequential HAIC with cisplatin powder and sorafenib improve the survival benefit compared with sorafenib alone as an initial therapy for advanced HCC. Since the one-year survival rate, a primary end point of the study, tended to be lower in the HAIC group than in the sorafenib group, our results suggest that sorafenib should be used as first-line therapy for advanced HCC. To the best of our knowledge, this is the first randomized study comparing an HAIC regimen versus sorafenib as an initial therapy for advanced $\mathrm{HCC}$.

HAIC has been frequently administered to patients with advanced HCC in Asian countries, especially those with PVTT [10-13]. Although its survival benefit remains unclear because of a lack of randomized control trials, a favorable disease control rate and tolerability were reported with variable regimens, including cisplatin plus low-dose 5-FU $[10,11], 5-\mathrm{FU}$ plus interferon $[12,13]$, and monotherapy 


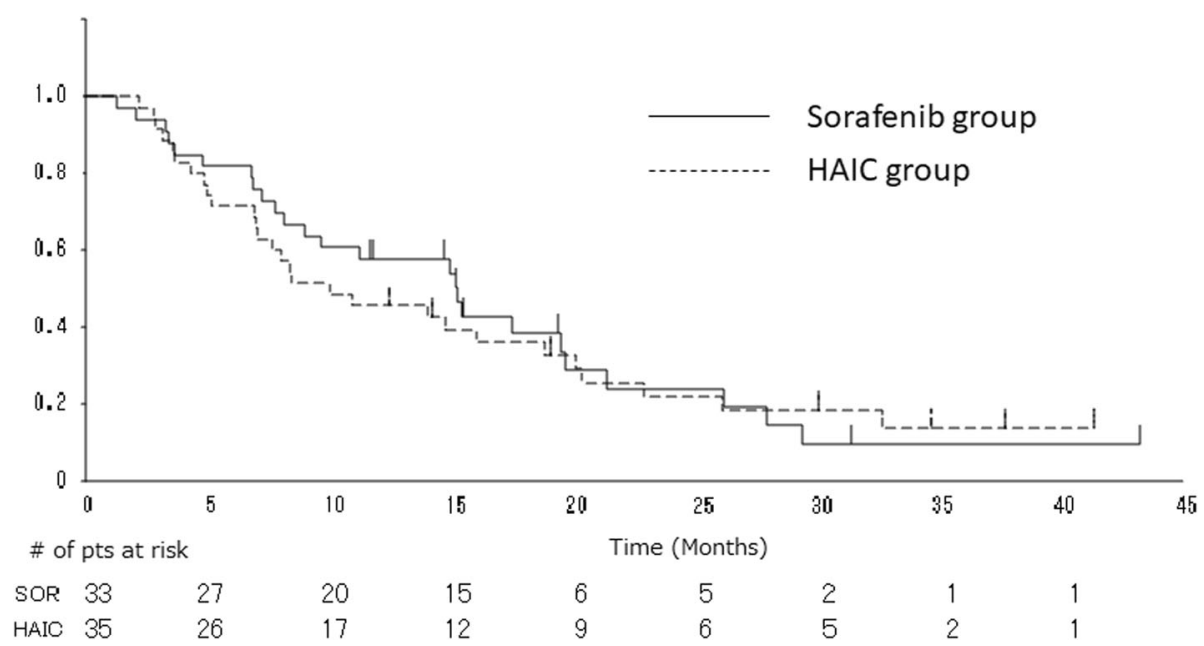

Fig. 1 Comparison of overall survival between the sorafenib group (solid line) and the HAIC group (dotted line). HAIC, hepatic arterial infusion chemotherapy

with cisplatin powder [14]. The outcome in a nationwide survey in Japan seems favorable [15], with a reported response rate of $45.9 \%$ and a disease control rate of $76.5 \%$. Advanced HCC patients who respond to HAIC have a prolonged OS, with a reported median overall survival (MST) of 40.7 months in responders to low-dose 5-FU and cisplatin [11]. We previously reported the efficacy of HAIC with cisplatin powder for advanced HCC with PVTT, and the MST of the responders was 37.3 months [18]. Thus, we planned to encourage the positioning of HAIC in the treatment of advanced HCC and conducted a multi-center randomized phase II study comparing sequential HAIC treatment using cisplatin powder followed by sorafenib versus sorafenib alone-a global standard option-as an initial therapy for advanced HCC.
Sorafenib, acting on the inhibition of a multikinase involved in tumor cell signaling, proliferation, angiogenesis, and apoptosis, is the only proven, global standard treatment for advanced HCC patients [20]. Sorafenib treatment in two prospective post-marketing analyses for Japanese patients $[7,8]$ was well tolerated; however, the discontinuation rate because of drug-related adverse events (AEs) was over $40 \%$ in both studies despite a reduction in the median daily dose of sorafenib from $614 \mathrm{mg}$ to $419 \mathrm{mg}$. A high discontinuation rate because of intolerance, particularly in elderly patients, has been reported $[9,21]$. In the present study, the ORR tended to be lower in the sorafenib group than in the HAIC group; however, the median TTP and OS tended to be higher in the sorafenib group than in the HAIC group. Subgroup analyses of OS according to the pretreatment

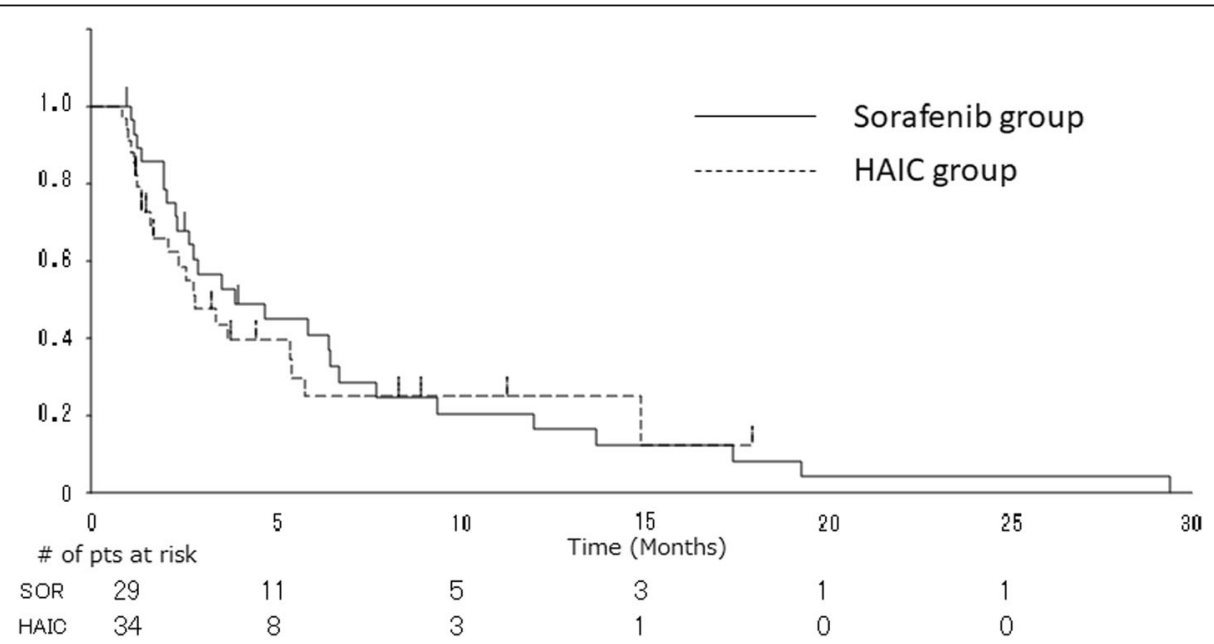

Fig. 2 Comparison of time to progression between the sorafenib group (solid line) and the HAIC group (dotted line). HAIC, hepatic arterial infusion chemotherapy 


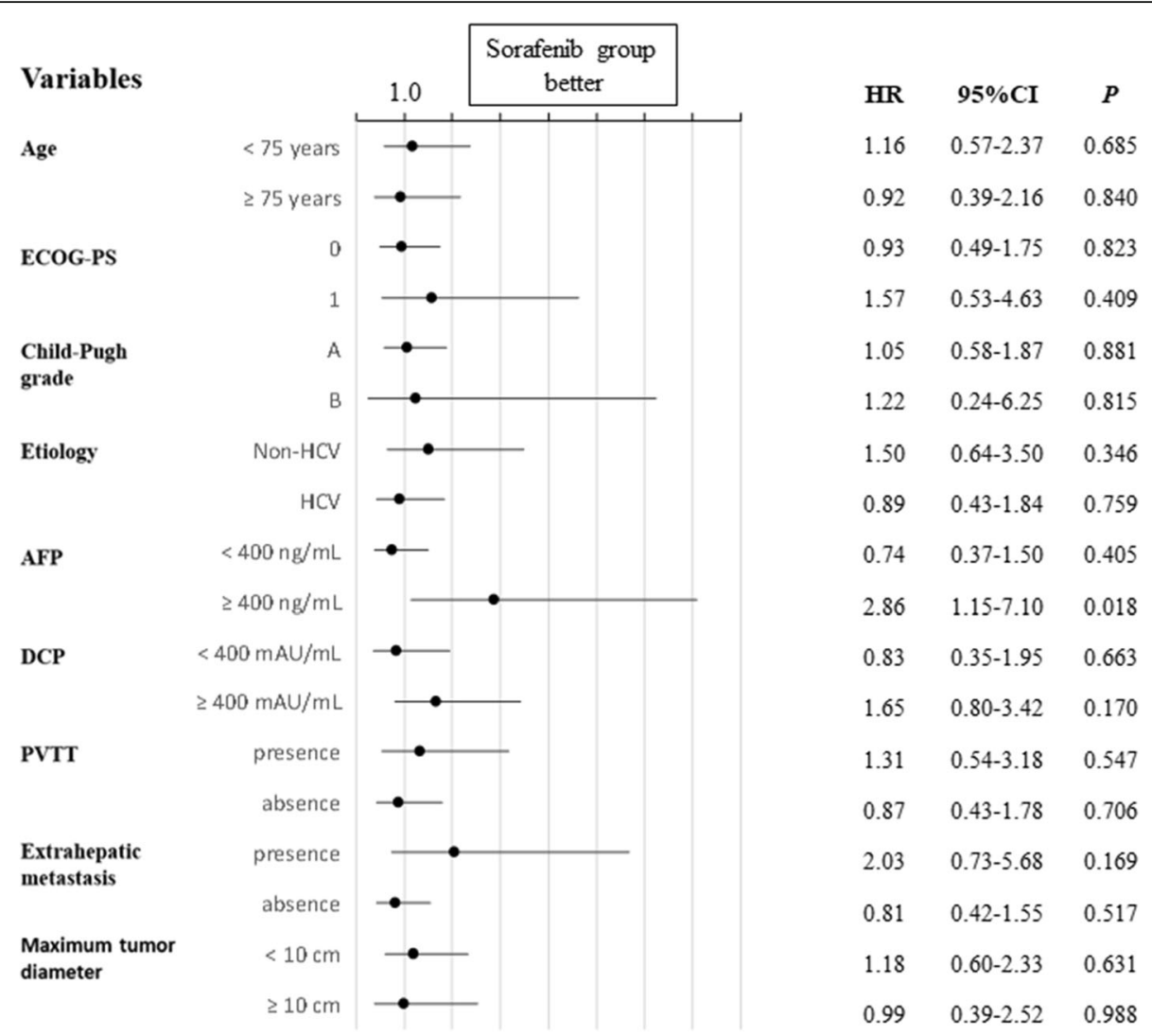

Fig. 3 Forest plots showing a subgroup analysis of overall survival. ECOG-PS, Eastern Cooperative Oncology Group-Performance Status; HCV, hepatitis C virus; AFP, alpha-fetoprotein; DCP, des-gamma carboxy-prothrombin; PVTT, portal vein tumor thrombosis. The HRs were calculated by univariate cox regression analysis

characteristics did not show any superiorities of HAIC to sorafenib, but sorafenib showed a favorable HR compared with HAIC in the subset of patients with a baseline AFP $\geq 400 \mathrm{ng} / \mathrm{mL}$, suggesting that sorafenib should be used as a first-line therapy for advanced HCC.

Patients with extrahepatic metastasis, irrespective of the prognosis, were eligible for inclusion in this study. In our clinical practice, the prognosis in patients with uncontrolled intrahepatic lesions seemed not to be affected by small extrahepatic metastases in the lymph nodes, lungs, or adrenal glands. Some studies have reported that effective treatments for intrahepatic lesions are of benefit to HCC patients with extrahepatic metastasis $[22,23]$. Although it is difficult to arrive at any conclusion regarding the efficacy of the treatment in patients with extrahepatic metastasis from this study because of the small sample size, there was no significant difference in the TTP between the two groups $(p=0.945)$.

Some reasons why sequential HAIC with cisplatin powder and sorafenib did not improve the survival benefit compared with sorafenib alone as an initial therapy for advanced HCC may be as follows: 1) the effectiveness of post-protocol treatments after sorafenib failure may have prolonged the post-progression survival (PPS), especially in the sorafenib group; and 2) the relatively high rate of protocol treatment discontinuation after HAIC-failure may have resulted in a loss of appreciation of the antitumor effect of the subsequent sorafenib treatment in the HAIC group. Regarding the former possibility, $88 \%$ of the patients in the sorafenib group received post-protocol treatment options, including HAIC with cisplatin powder which was administered in $55 \%$ of the patients of the sorafenib group. Therefore, crossover of the treatment options, including HAIC and sorafenib, could minimize the difference in the OS between the two groups. Since HAIC using low-dose 5-FU and cisplatin has been shown to exhibit moderate antitumor efficacy for advanced HCC patients after sorafenib failure [24], these post-protocol treatment options may prolong the PPS after sorafenib failure, thereby contributing to a longer OS in the sorafenib group [25, 26]. Regarding the latter possibility, 8 (23\%) of the 35 patients did not start subsequent sorafenib treatment after HAIC-failure because of a deterioration in their general conditions, a decrease in hepatic functional reserve, and drug-related 
Table 3 Serious adverse events due to protocol treatment in the sorafenib group and the HAIC group

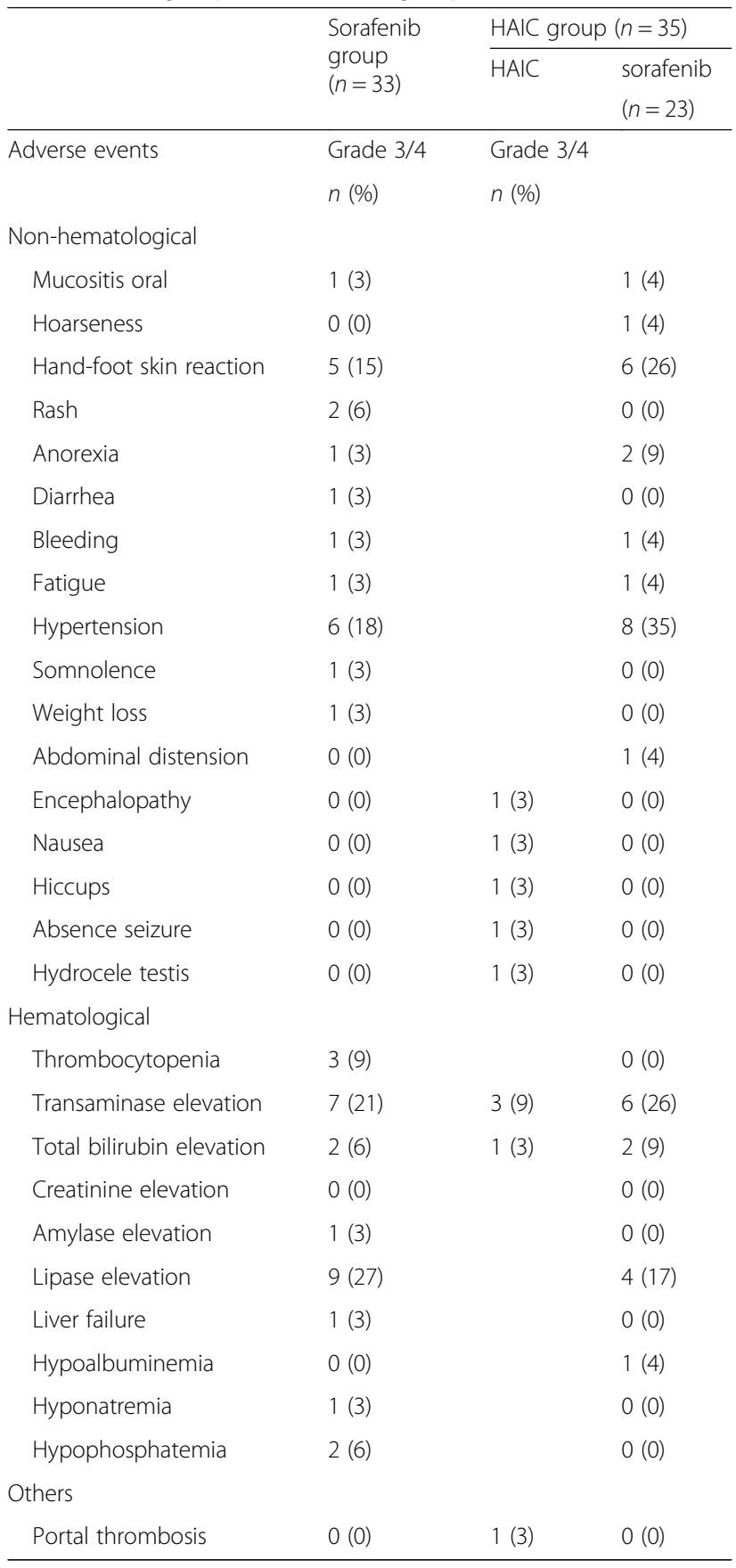

HAIC hepatic arterial infusion chemotherapy

toxicities, potentially losing any advantage of sorafenib treatment in the HAIC group.

Because HCC has heterogeneous intratumor characteristics $[27,28]$, multidisciplinary treatments, including combination or sequential treatments, might have some impact on the treatment of advanced HCC patients. In our sequential setting, HAIC with cisplatin powder followed by sorafenib did not improve the survival
Table 4 Post protocol treatment in the sorafenib group and the HAIC group

\begin{tabular}{lll}
\hline Post protocol treatment & Sorafenib group (\%) & HAIC group (\%) \\
\hline Absent & $4(12)$ & $17(49)$ \\
Present & $29(88)$ & $18(51)$ \\
sorafenib continuation & $11(33)$ & $10(29)$ \\
Resection & $0(0)$ & $1^{\mathrm{a}}(3)$ \\
RFA & $2(6)$ & $1^{\mathrm{b}}(3)$ \\
TACE & $5(15)$ & $5^{\mathrm{c}}(14)$ \\
HAIC & $18(55)$ & $3(9)$ \\
Others & $1^{\mathrm{d}}(3)$ & $2^{\mathrm{e}}(6)$
\end{tabular}

HAIC hepatic arterial infusion chemotherapy, RFA radiofrequency ablation, TACE transarterial chemoembolization; Resection as a conversion option were administered in one patient ${ }^{a}$, RFA in one ${ }^{b}$, and TACE in two of five patients ${ }^{c}$. d, One patient participated in a clinical trial examining tivantinib; ${ }^{\mathrm{e}}$, One patient participated in a clinical trial examining tivantinib and another one received percutaneous ethanol injection treatment (PEIT)

benefit compared with sorafenib alone as an initial therapy for advanced HCC. Regarding combined settings, two prospective randomized controlled trials of sorafenib plus HAIC using low-dose 5-FU and cisplatin (lowdose FP therapy) versus sorafenib alone (SILIUS study: NCT01214343) [29] and of sorafenib plus HAIC using cisplatin powder versus sorafenib alone (CDDP-Sorrandomized Phase II [rP2] study: UMIN000005703) [30] for patients with advanced HCC have been recently reported. In the SILIUS study, the addition of HAIC to sorafenib alone did not have any effect on the OS in this Phase III trial [29], and a superior OS was limited to the subgroup of patients with main portal vein invasion. In the CDDP-Sor-rP2 study, a favorable effect on OS has been reported for the addition of HAIC using cisplatin powder to sorafenib [30]; the stratified HR (95\% CI) was $0.60(0.38-0.96)(P=0.031)$, and a Phase III trial is anticipated.

The present study had some limitations; it was mainly attributable to the difficulty in recruiting suitable patients, and the number of enrolled patients was less than that required by the study design. Our HCC patients could select any of the following treatment regimens: 1) sorafenib treatment, 2) HAIC with other chemotherapeutic agents, or 3) participation in other clinical trials involving the use of other multikinase inhibitors or anticancer agents. With regard to HAIC with other regimens, we do not use any of the other commonly used agents for HAIC in Japan, such as low-dose FP therapy or 5-FU plus interferon- $\alpha$ (FAIT therapy). A phase II study comparing the efficacy of FAIT therapy to those of low-dose FP therapy or intraarterial cisplatin as a reference group did not show any efficacious differences between the treatment groups [31], suggesting that intraarterial cisplatin powder may be comparable to lowdose FP therapy or FAIT therapy. Another limitation 
was that the HAIC could be halted in the event of clinical PD, based on our personal communications. In the present study, the second session of HAIC could be avoided in 7 patients and the treatment was switched to sorafenib after the first session of HAIC. The median OS of these 7 patients was 10.9 months (2.8-15.8 months), which was comparable to the median OS (10 months) of all patients in the HAIC group, suggesting that our definition of "clinical PD" is unlikely to be disadvantageous for our patients.

\section{Conclusions}

A survival benefit of HAIC with cisplatin powder followed by sorafenib was not demonstrated. Further studies on determining an appropriate treatment regimen in HAIC may be important, including a combination setting with sorafenib or a sequential setting after sorafenib failure.

\section{Abbreviations \\ AEs: Adverse events; AFP: Alpha-fetoprotein; BCLC: Barcelona Clinic Liver Cancer; Cl: Confidence interval; CR: Complete response; CT: Computed tomography; CTCAE: Common Terminology Criteria for Adverse Events; DCP: Des-gamma carboxyprothrombin; DCR: Disease control rate; ECOG: Eastern Cooperative Oncology Group; HAIC: Hepatic arterial infusion chemotherapy; HCC: Hepatocellular carcinoma; HR: Hazard ratio; mRECIST: modified Response Evaluation Criteria for Solid Tumors: MRI: Magnetic resonance imaging; MST: Median overall survival; ORR: Objective response rate; OS: Overall survival; PD: Progressive disease; PPS: Post-progression survival; PVTT: Portal vein tumor thrombosis; RFA: Radiofrequency ablation; TACE: Transarterial chemoembolization; TTP: Time to progression}

\section{Acknowledgements}

Not applicable.

\section{Authors' contributions}

$M K, K T$, and MT contributed to the design of the study, the preparation and analysis of the datasets, and statistical analysis. TI contributed the preparation of the datasets and carried out the data entry. MK, MM, SK, SO, HH, TN, HA, $\mathrm{TH}, \mathrm{DT}, \mathrm{KM}, \mathrm{CO}, \mathrm{MS}, \mathrm{KN}, \mathrm{SM}$, and $\mathrm{KT}$ participated in the preparation of the patient inclusion, treatment, and follow-up workflow. All authors carefully read and approved this manuscript.

\section{Funding}

This work was supported by the Waksman Foundation of Japan INC.

\section{Availability of data and materials}

The datasets generated and/or analysed during the current study are not publicly available because it was not a big project study to have such a public datasets, but are available from the corresponding author on reasonable request.

\section{Ethics approval and consent to participate}

This study was performed according to the guidelines of the Helsinki Declaration

This trial was reviewed and approved by the review board of Yokohama City University Medical Center on 22 July 2011: registration number D1 10721005. This was approved by the review board of all participating institutes which are:

Kitasato University Hospital on 19 October 2011: registration number 11-696. St Marianna University School of Medicine on 24 November 2011: registration number 2003.

St Marianna University School of Medicine, Yokohama City Seibu Hospital on 24 November 2011: registration number 2003.
Kawasaki Municipal Tama Hospital, Yokohama City Seibu Hospital on 24 November 2011: registration number 2003.

Kanagawa Cancer Center Hospital on 7 December 2011: registration number 41.

Hiroshima University on 5 December 2012: registration number 363.

Isesaki Municipal Hospital on 26 September 2013.

Written informed consent was obtained from all the patients.

\section{Consent for publication}

Not applicable

\section{Competing interests}

Morimoto received honoraria from Bayer and Okuse received honoraria from AbbVie. The other authors declare that they have no competing interests.

\section{Author details}

${ }^{1}$ Gastroenterological Center, Yokohama City University Medical Center 4-57, Urafune-cho, Minami-ku, Yokohama, Kanagawa 232-0024, Japan.

${ }^{2}$ Department of Gastroenterology, Yokohama City University Hospital; 3-9, Fukuura, Kanazawa-ku, Yokohama, Kanagawa 236-0004, Japan. ${ }^{3}$ Hepatobiliary and Pancreatic Medical Oncology, Kanagawa Cancer Center Hospital; 1-1-2, Nakao, Asahi-ku, Yokohama, Kanagawa 241-0815, Japan. ${ }^{4}$ Gastroenterology Division of Internal Medicine, Kitasato University Hospital; 1-15-1, Kitasato, Minami-ku, Sagamihara, Kanagawa 252-0329, Japan. ${ }^{5}$ Department of Gastroenterology and Metabolism, Applied Life Sciences, Institute of Biomedical and Health Sciences, Hiroshima University; 1-2-3, Kasumi, Minami-ku, Hiroshima, Hiroshima 734-8551, Japan. ${ }^{6}$ Department of Internal Medicine, Isesaki Municipal Hospital; 12-1, Tsunatorihonmachi, Isesaki, Gunma 372-0817, Japan. ${ }^{7}$ Division of Gastroenterology and Hepatology, Department of Internal Medicine, St Marianna University School of Medicine; 2-16-1, Sugao, Miyamae-ku, Kawasaki, Kanagawa 216-8511, Japan. ${ }^{8}$ Division of Gastroenterology and Hepatology, Kawasaki Municipal Tama Hospital; 1-30-37, Shukugawara, Tama-ku, Kawasaki, Kanagawa 214-8525, Japan. ${ }^{9}$ Department of Data Science, Yokohama City University School of Data Science; 3-9, Fukuura, Kanazawa-ku, Yokohama, Kanagawa 236-0004, Japan.

${ }^{10}$ Yokohama City University Center for Novel and Exploratory Clinical trials;

1-1-1, Fukuura, Kanazawa-ku, Yokohama, Kanagawa 236-0004, Japan.

${ }^{11}$ Gastroenterology Division, Hadano Red Cross Hospital; 1-1-1, Tatenodai, Hadano, Kanagawa 257-0017, Japan

Received: 21 March 2019 Accepted: 24 September 2019

Published online: 15 October 2019

\section{References}

1. Forner A, Llovet JM, Bruix J. Hepatocellular carcinoma. Lancet. 2012;379: 1245-55.

2. Grieco A, Pompili M, Caminiti G, Miele L, Covino M, Alfei B, et al. Prognostic factors for survival in patients with early-intermediate hepatocellular carcinoma undergoing non-surgical therapy: comparison of Okuda, CLIP, and BCLC staging systems in a single Italian Centre. Gut. 2005;54:411-8.

3. Llovet JM, Di Bisceglie AM, Bruix J, Kramer BS, Lencioni R, Zhu AX, et al. Panel of experts in HCC-design clinical trials. Design and endpoints of clinical trials in hepatocellular carcinoma. J Natl Cancer Inst. 2008:100:698-711.

4. Llovet JM, Ricci S, Mazzaferro V, Hilgard P, Gane E, Blanc JF, et al. Sorafenib in advanced hepatocellular carcinoma. N Engl J Med. 2008;359:378-90.

5. Cheng AL, Kang YK, Chen Z, Tsao CJ, Qin S, Kim JS, et al. Efficacy and safety of sorafenib in patients in the Asia-Pacific region with advanced hepatocellular carcinoma: a phase III randomized, double-blind, placebocontrolled trial. Lancet Oncol. 2009:10:25-34.

6. Bruix J. ShermanM; American association for the study of liver diseases. Management of hepatocellular carcinoma: an update. Hepatology. 2011; 53:1020-2.

7. Kaneko S, Ikeda K, Matsuzaki Y, Furuse J, Minami H, Okayama Y, et al. Safety and effectiveness of sorafenib in Japanese patients with hepatocellular carcinoma in daily medical practice: interim analysis of a prospective postmarketing all-patient surveillance study. J Gastroenterol. 2016:51:1011-21.

8. Kudo M, Ikeda M, Takayama T, Numata K, Izumi N, Furuse J, et al. Safety and efficacy of sorafenib in Japanese patients with hepatocellular 
carcinoma in clinical practice: a subgroup analysis of GIDEON. J Gastroenterol. 2016;51:1150-60.

9. Morimoto M, Numata K, Kondo M, Hidaka H, Takada J, Shibuya A, et al. Higher discontinuation and lower survival rates are likely elderly Japanese patients with advanced hepatocellular carcinoma receiving sorafenib. Hepatol Res. 2011:41:296-302.

10. Ando E, Tanaka M, Yamashita F, Kuromatsu R, Yutani S, Fukumori K, et al. Hepatic arterial infusion chemotherapy for advanced hepatocellular carcinoma with portal vein tumor thrombosis: analysis of 48 cases. Cancer. 2002:95:588-95.

11. Ueshima K, Kudo M, Takita M, Nagai T, Tatsumi C, Ueda T, et al. Hepatic arterial infusion chemotherapy using low-dose 5 -fluouracil and cisplatin for advanced hepatocellular carcinoma. Oncology. 2010;78:1148-53.

12. Sakon M, Nagano H, Dono K, Nakamori S, Umeshita K, Yamada A, et al. Combined intraarterial 5-fluorouracil and subcutaneous interferon-alpha therapy for advanced hepatocellular carcinoma with tumor thrombi in the major portal branches. Cancer. 2002:94:435-42.

13. Obi S, Yoshida H, Toune R, Unuma T, Kanda M, Sato S, et al. Combination therapy of intraarterial 5 -fluouracil and systemic interferon-alpha for advanced hepatocellular carcinoma with portal venous invasion. Cancer. 2006;106:1990-7.

14. Yoshikawa M, Ono N, Yodono H, Ichida T, Nakamura H. Phase II study of hepatic arterial infusion of a fine-powder formulation of cisplatin for advanced hepatocellular carcinoma. Hepatol Res. 2008;38:478-83.

15. Kudo M. Treatment of advanced hepatocellular carcinoma with emphasis on hepatic arterial infusion chemotherapy and molecular targeted therapy. Liver Cancer. 2012;1:62-70.

16. Court WS, Order SE, Siegel JA, Johnson E, DeNittis AS, Principato R, et al. Remission and survival following monthly intraarterial cisplatinum in nonresectable hepatoma. Cancer Investig. 2002;20(5-6):613-25.

17. Seldinger SI. Catheter replacement of the needle in percutaneous arteriography. Acta Radiol. 1952;39:368-76

18. Kondo M, Morimoto M, Numata K, Nozaki A, Tanaka K. Hepatic arterial infusion therapy with fine powder formulation of cisplatin for advanced hepatocellular carcinoma with portal vein tumor thrombosis. Jpn J Clin Oncol. 2011;41:69-75.

19. Lencioni R, Llovet JM. Modified RECIST (mRECIST) assessment for hepatocellular carcinoma. Semin Liver Dis. 2010;30:52-60.

20. Adnane L, Trail PA, Taylor I, Wilhelm SM. Sorafenib (BAY 43-9006, Nexavar ${ }^{\circledR}$ ), a dual-action inhibitor that targets RAF/MEK/ERK pathway in tumor cells and tyrosine kinases VEGFR/PDGFR in tumor vasculature. Methods Enzymol. 2006;407:597-612.

21. Williet N, Clavel L, Bourmaud A, Verot C, Bouarioua N, Roblin X, et al. Tolerance and outcomes of sorafenib in elderly patients treated for advanced hepatocellular carcinoma. Dig Liver Dis. 2017;49:1043-9.

22. Uchino K, Tateishi R, Shiina S, Kanda M, Masuzaki R, Kondo $Y$, et al. Hepatocellular carcinoma with extrahepatic metastasis. Cancer. 2011; 117:4475-83.

23. Xia F, Wu L, Lau W-Y, Li G, Huan H, Qian C, et al. Positive lymph node metastasis has a marked impact on the long-term survival of patients with hepatocellular carcinoma with extrahepatic metastasis. PLoS ONE. 9(4):e95889.

24. Terashima T, Yamashita T, Arai K, Sunagozaka H, Kitahara M, Nagaoka $H_{4}$ et al. Feasibility and efficacy of hepatic arterial infusion chemotherapy for advanced hepatocellular carcinoma after sorafenib. Hepatol Res. 2014:44:1179-85.

25. Terashima T, Yamashita T, Tanaka N, Nakagawa H, Toyama T, Arai K, et al. Post-progression survival and progression-free survival in patients with advanced hepatocellular carcinoma treated by sorafenib. Hepatol Res. 2016;46:650-6.

26. Kondo M, Numata K, Hara K, Nozaki A, Fukuda H, Chuma M, et al. Treatment of advanced hepatocellular carcinoma after failure of sorafenib treatment: subsequent or additional treatment interventions contribute to prolonged survival post-progression. Gastroenterol Res Pract. 2017. https://doi.org/10. 1155/2017/5728946.

27. Villanueva A, Llovet JM. Impact of intra-individual molecular heterogeneity in personalized treatment of hepatocellular carcinoma. Hepatology. 2012;56:2416-9.

28. Llovet JM, Villanueva A, Lachenmayer A, Finn RS. Advances in targeted therapies for hepatocellular carcinoma in the genomic era. Nat Rev Clin Oncol. 2015;12:408-24.
29. Kudo M, Ueshima K, Yokosuka O, Obi S, Izumi N, Aikata H, et al. Prospective randomized controlled phase III trial comparing the efficacy of sorafenib versus sorafenib in combination with low-dose cisplatin/fluorouracil hepatic arterial infusion chemotherapy in patients with advanced hepatocellular carcinoma. J Hepatol. 2016;64:S183-212.

30. Ikeda M, Shimizu S, Sato T, Morimoto M, Kojima Y, Inaba Y, et al. Sorafenib plus hepatic arterial infusion chemotherapy with cisplatin versus sorafenib for advanced hepatocellular carcinoma: randomized phase II trial. Ann Oncol. 2016;27:2090-6.

31. Monden M, Sakon M, Sakata Y, Ueda Y, Hashimura E, FAIT Research Group. 5-fluorouracil arterial infusion + interferon therapy for highly advanced hepatocellular carcinoma: a multicenter, randomized, phase II study. Hepatol Res. 2012;42:150-65.

\section{Publisher's Note}

Springer Nature remains neutral with regard to jurisdictional claims in published maps and institutional affiliations.

Ready to submit your research? Choose BMC and benefit from:

- fast, convenient online submission

- thorough peer review by experienced researchers in your field

- rapid publication on acceptance

- support for research data, including large and complex data types

- gold Open Access which fosters wider collaboration and increased citations

- maximum visibility for your research: over $100 \mathrm{M}$ website views per year

At BMC, research is always in progress.

Learn more biomedcentral.com/submissions 